\title{
A Fault Diagnosis Approach for Urban Railway Gearbox Based on EMD Method and Elman Neural Network
}

\author{
Wang Xing ${ }^{1, *}$, Qi Xiangdong ${ }^{1}$, Li Baojin ${ }^{2}$ and Skopincev E. Vektorovich ${ }^{3}$ \\ ${ }^{I}$ Taiyuan University of Science and Technology, Taiyuan Shanxi, 030024, P.R. China \\ ${ }^{2}$ Taiyuan Tong xin de Tech. \& Trading Co., Ltd, Taiyuan Shanxi, 030024, P.R. China \\ ${ }^{3}$ Moscow State University of Mechanical Engineering, Bolshaya Semenovskaya str., Moscow
}

\begin{abstract}
As for the non-linearity and non-stationary characteristics of the vibration signals of urban railway gearbox, an efficient method for gearbox fault detection and diagnosis based on EMD (empirical mode decomposition) and Elman neural network is proposed. First of all, the original signals are decomposed into a number of IMFs (intrinsic mode function) by EMD. Secondly, the feature vectors are constructed. Finally, these eigenvectors as fault samples input to the Elman neural network. The recognition results show that the EMD and Elman neural network is effective in railway gearbox fault diagnosis. This approach can be used as a useful tool for the rotating machinery fault diagnosis.
\end{abstract}

Keywords: Urban railway gearbox, EMD, Elman neural network, IMF, fault diagnosis.

\section{INTRODUCTION}

Gear is a key component of the rotating machinery, in the harsh working conditions of the most vulnerable subjected to progressive deterioration [1]. A failure of the gearbox may cause breakdown of the urban rail vehicle. Therefore, a practical fault diagnosis of gearbox is necessary to monitor the vehicle.

EMD is an adaptive signal processing method, and it is suitable for dealing with non-stationary and nonlinear signals. The decomposed components, which are called IMFs, can be determined from the signal characteristics. EMD is able to perform decomposition of the raw signal and automatically determine the level of decomposition based on the nature of that raw signal [2]. The neural network has a strong ability in nonlinear mapping and fault tolerance ability. Implementation of neural network nonlinear robust fault diagnosis is very easy. And Elman neural network have many more advantages than BP neural network, such as: faster convergence speed, less training iteration, stronger robustness and no local minimum...etc [3]. So this paper aims to propose an effective method for railway gearbox fault diagnosis by using MD and Elman neural network.

The arrangement of this paper are as follows. In section 2, we introduce the EMD method and the feature vector extraction algorithm. Section 3 defines the Elman neural network. In Section 4, Simulation results are proposed to demonstrate the effectiveness of the provided algorithm for faults diagnosis of urban railway gearbox. Finally, the Section 5 is the conclusions.

\section{EMD METHOD AND FEATURE VECTORS EX- TRACTION ALGORITHM}

\subsection{EMD Method}

EMD is an adaptive signal decomposition method, it can decompose non-linear and non-stationary data sequence into an AM/FM components or alike. These independent components can be obtained are called intrinsic mode functions, it must satisfy the two conditions: (1) In the whole data set, the number of extrema and the number of zero-crossings must be different at most by one. (2) At any point, by the local maxima and local minima defined envelope average to zero [4].

Specific algorithm refers to reference [5], the initial signals $x(t)$ after EMD treatment can be express as:

$x(t)=\sum_{j=1}^{n} c_{j}+r_{n}$

Where, $r_{n}$ is residual error function, represents average trend of signals; IMF components $c_{1}, c_{2}, \cdots, c_{n}$ contain different elements respectively from low to high frequency of signals.

\subsection{Feature Vectors Extraction Algorithm}

The steps of time-frequency domain feature extraction are as follows: 


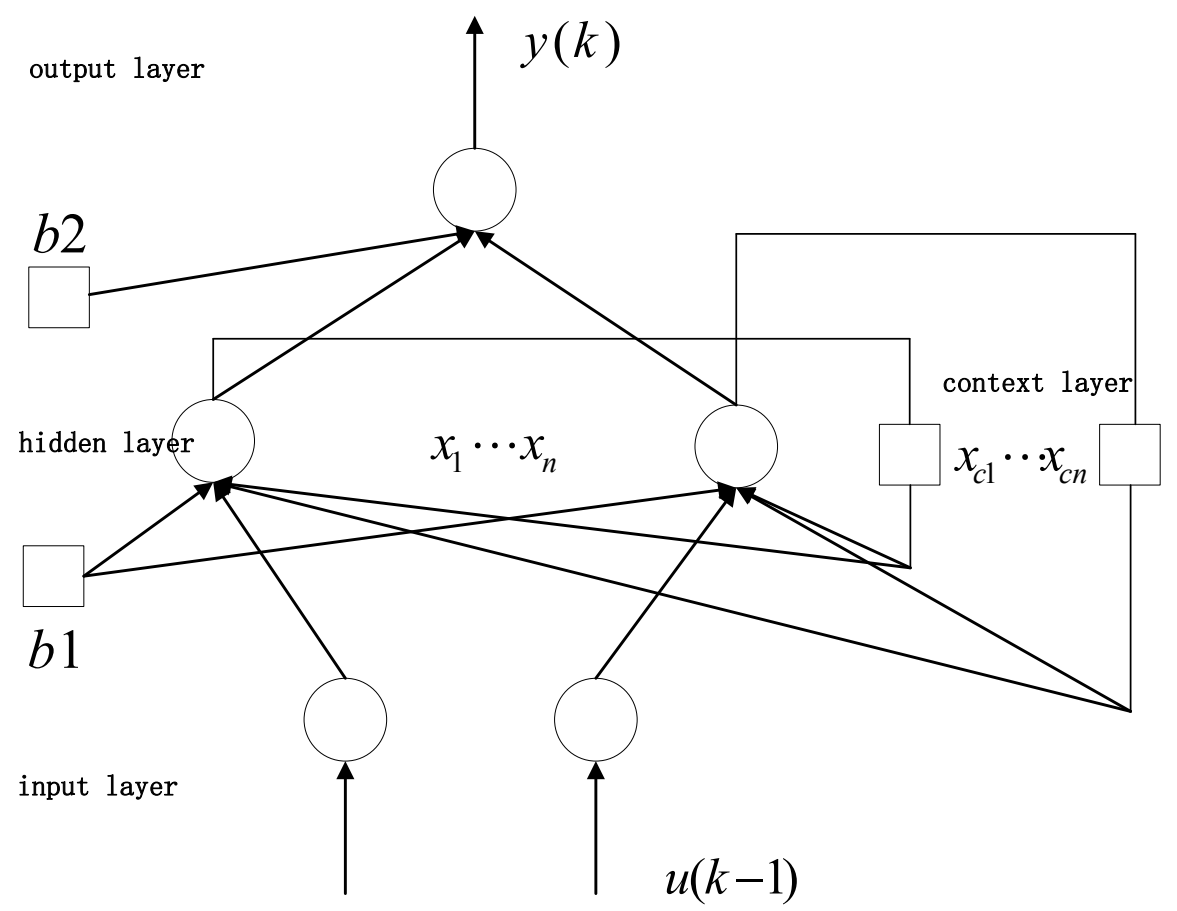

Fig. (1). Topology structure of Elman neural network.

(1) The vibration signals are broken into some IMFs by using the EMD method, the first $n$ IMFs $c_{i}(t)$, $i=1,2,3, \cdots, n$, which include the most dominant fault energy are chosen to extract the feature.

(2) Calculate the energy-torque of every small time block

The formula to calculate IMF energy-torque is:

$E_{i}=\int_{-\infty}^{+\infty} t\left|c_{i}(t)\right|^{2} d t$

For discrete signals, the formula to calculate energytorque is:

$E_{i}=\sum_{k=1}^{m}(k \cdot \Delta t)\left|c_{i}(k \cdot \Delta t)\right|^{2}$

Where $m$ is the total number of sampling points, $k$ is the sampling points, $\Delta t$ is the sampling period. Calculating the energy-torque $E_{1}, E_{2}, \cdots$ for each chosen IMF based on the formula (3).

(3) Constructing the feature vector $T$ in the elements of the energy-torque.

$T=\left[\begin{array}{llll}E_{1} & E_{2} & \cdots & E_{n}\end{array}\right]$

When the energy-torque is a larger numerical, normalizing $T$ and get the normalized feature vector $T^{\prime}$

Among them:
$E=\left(\sum_{i=1}^{n}\left|E_{i}\right|^{2}\right)^{\frac{1}{2}}$

The formula to calculate IMF energy-torque is [6]:

$$
E_{i}=\int_{-\infty}^{+\infty}\left|c_{i}(t)\right|^{2} d t
$$

\section{ELMAN NEURAL NETWORK MODEL}

The architecture of the Elman network which includes the input layer, the output layer, the hidden layer and the context layer is an extension of standard feedforward networks. The outputs context units and the external input neurons are input to the hidden layer neurons. Context units are also memory units as they store the previous output of hidden neurons. Therefore, the nonlinear dynamic system of Elman neural network with dynamic memory function contributes to the observed, improve storage stability $[7,8]$. We express the network as follows:

$$
\begin{aligned}
& x(k)=f\left(w^{1} x_{c}(k)+w^{2}(u(k-1))\right) \\
& x_{c}(k)=x(k-1) \\
& y(k)=g\left(w^{3} x(k)\right)
\end{aligned}
$$

Where $W^{1}, W^{2}, W^{3}$ are the weight matrices of context units to hidden units, input units to hidden units and hidden units to output units respectively. $f(\cdot)$ and $g(\cdot)$ are 


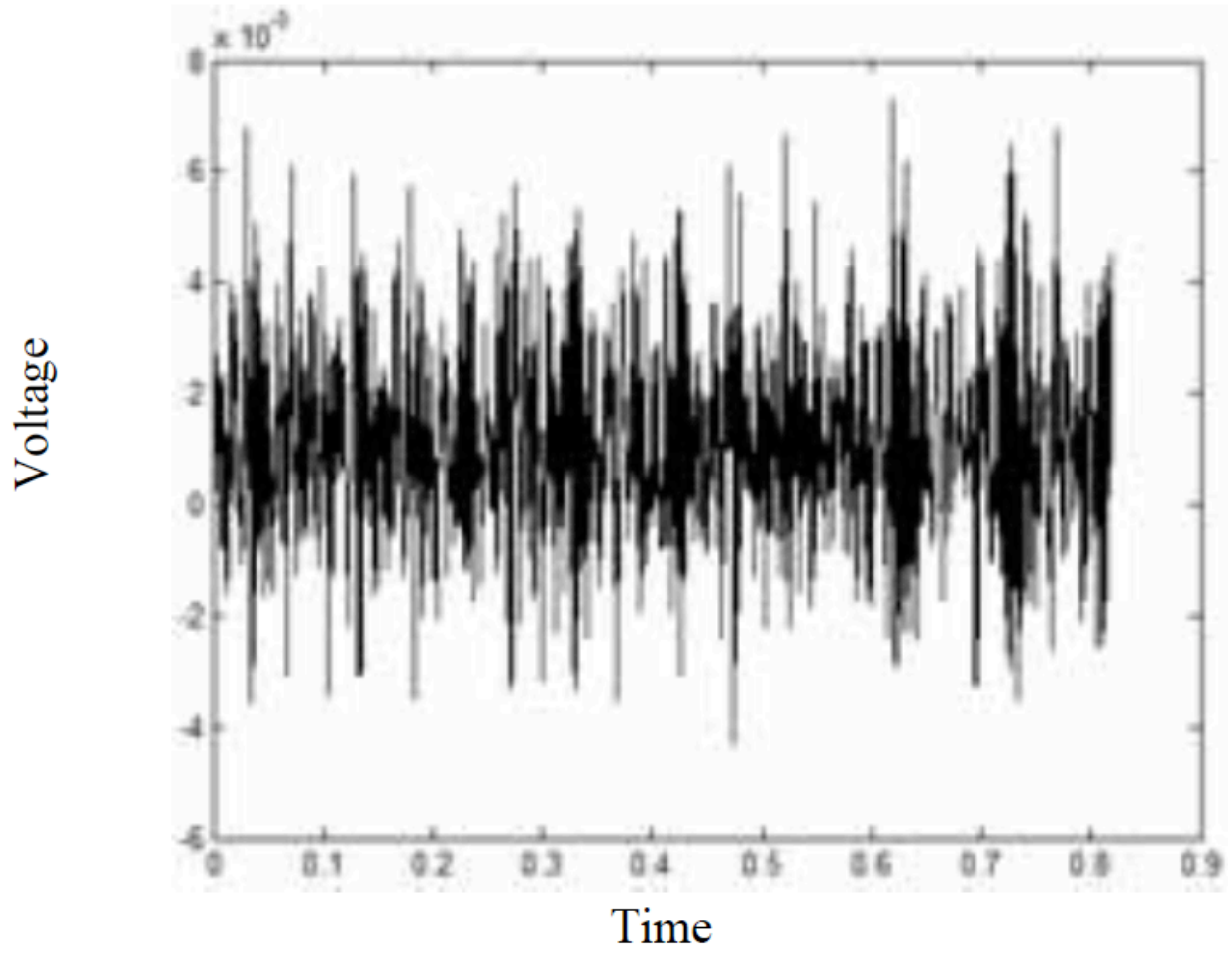

Fig. (2). The time domain of normal signal.

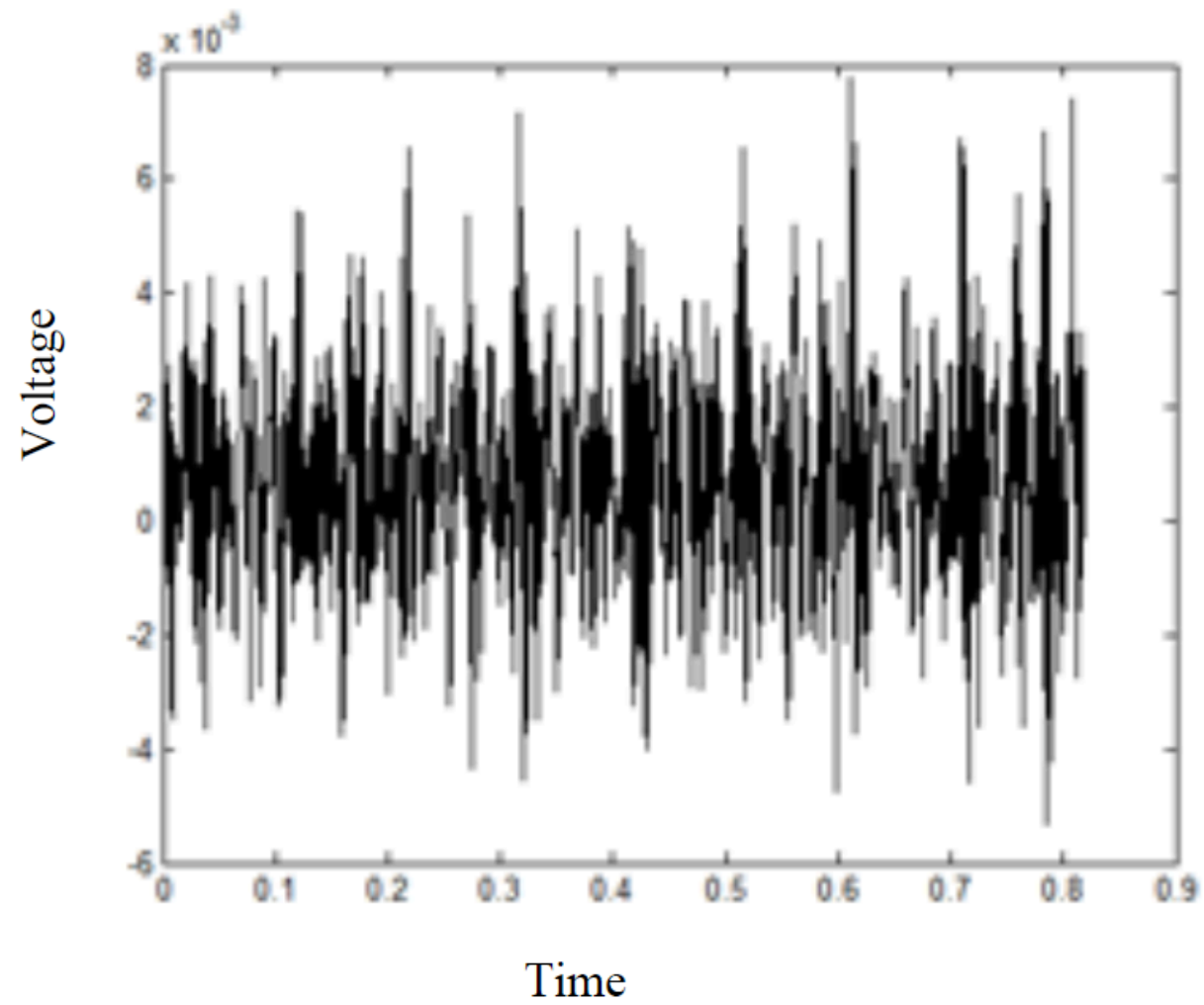

Fig. (3). The time domain of tooth wear signal. 


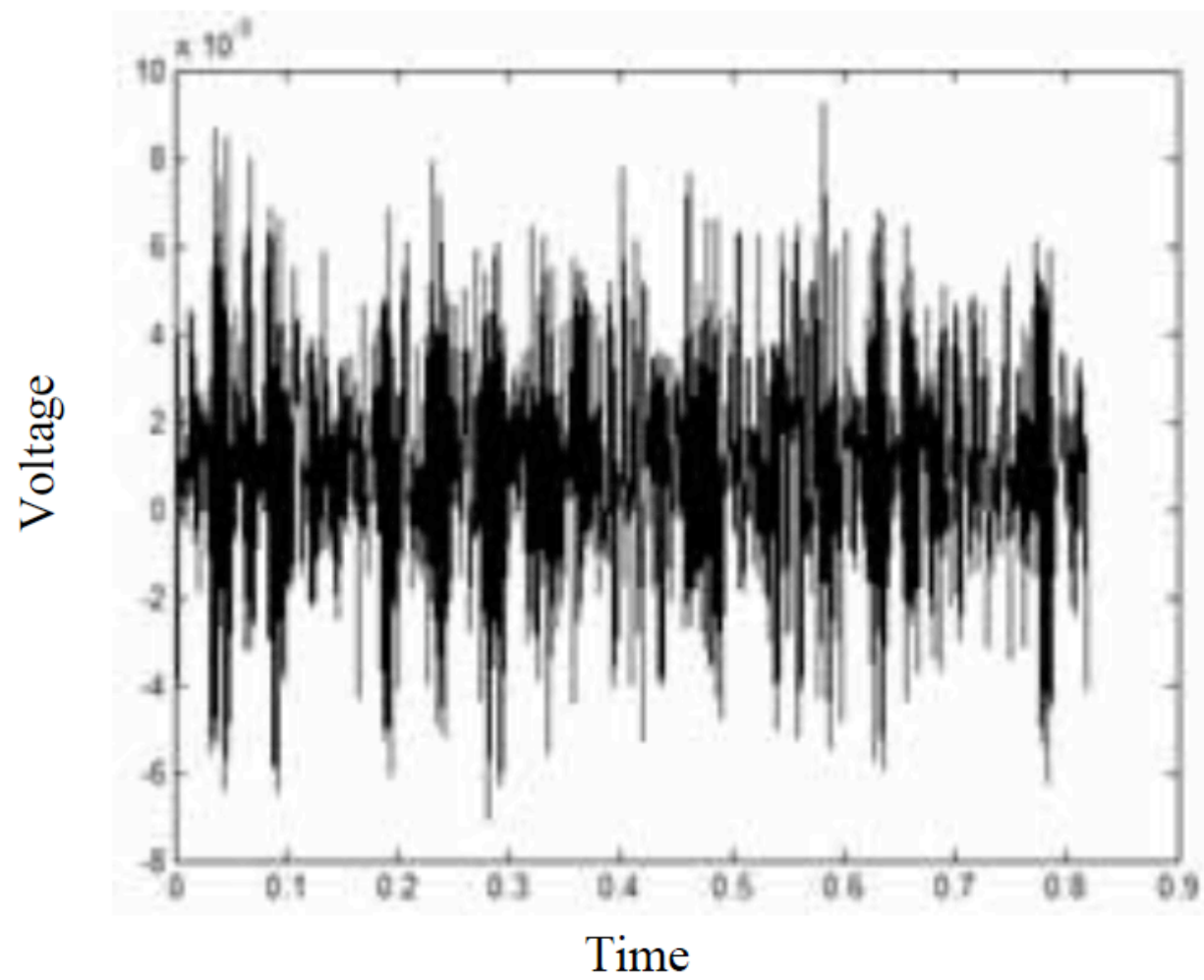

Fig. (4). The time domain of tooth break signal.
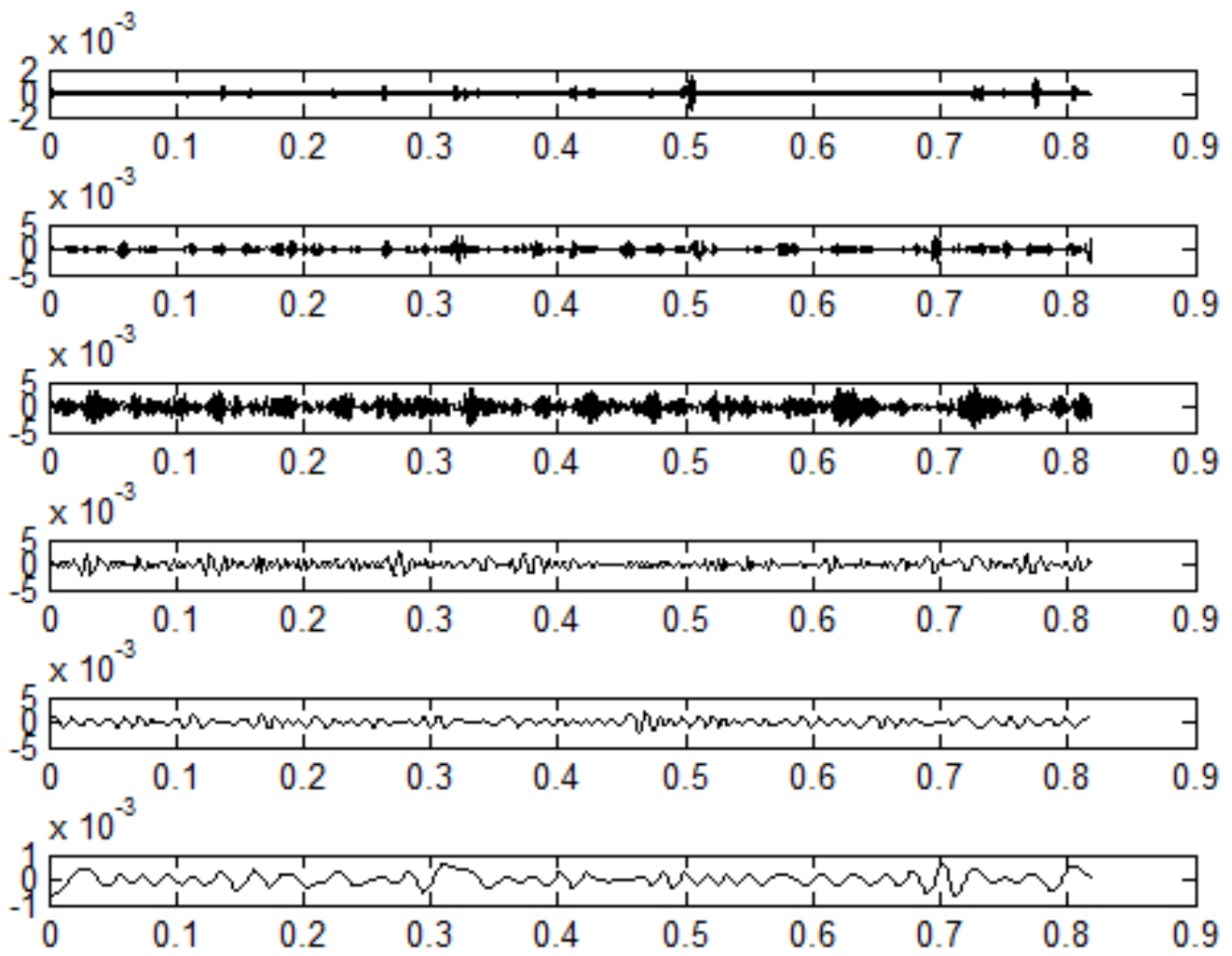

Fig. (5). Empirical mode decomposition of the normal signal. 

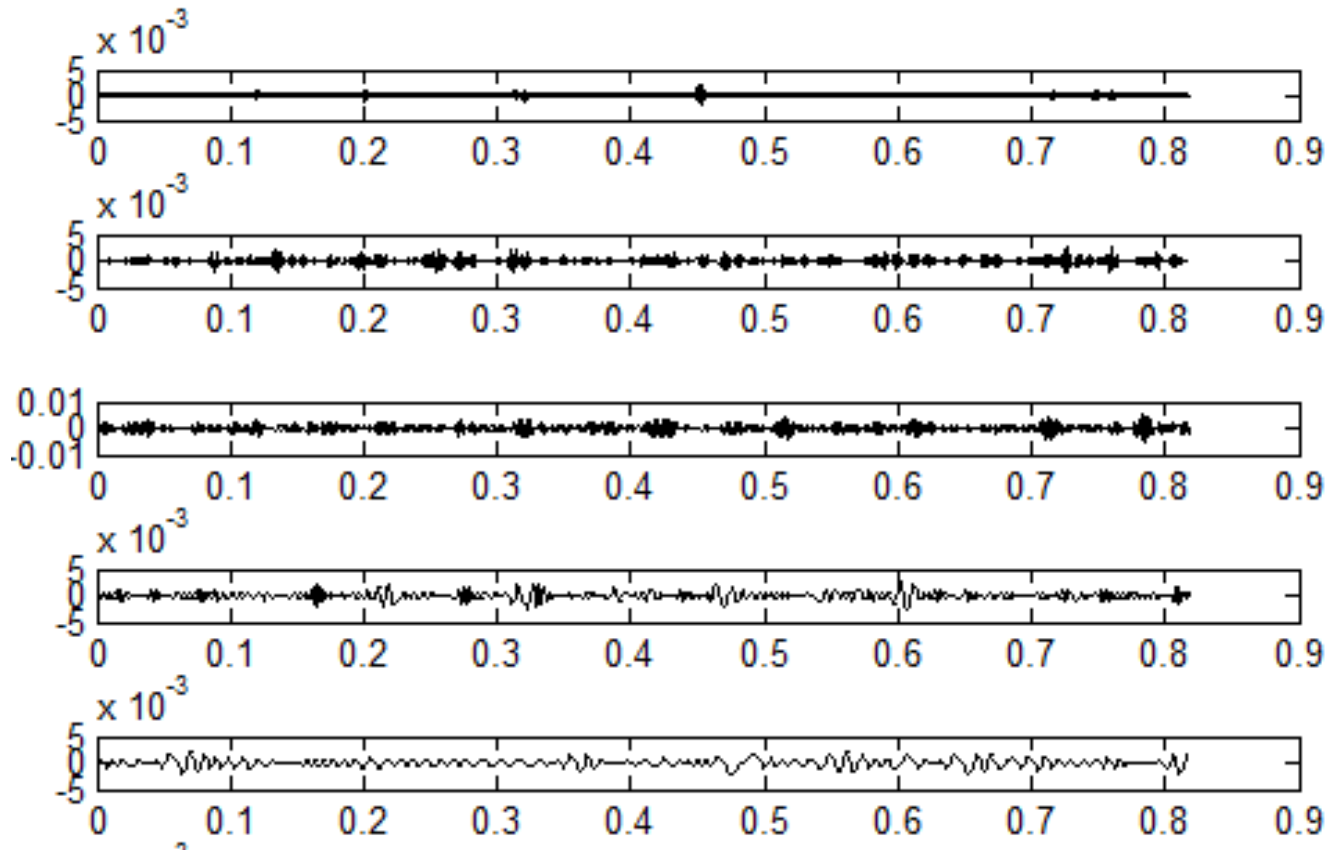

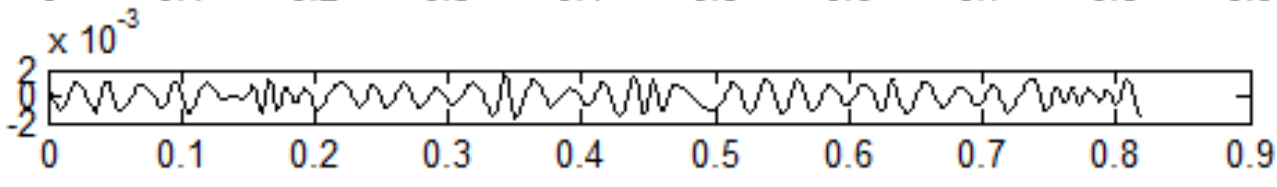

Fig. (6). Empirical mode decomposition of the tooth wear signal.
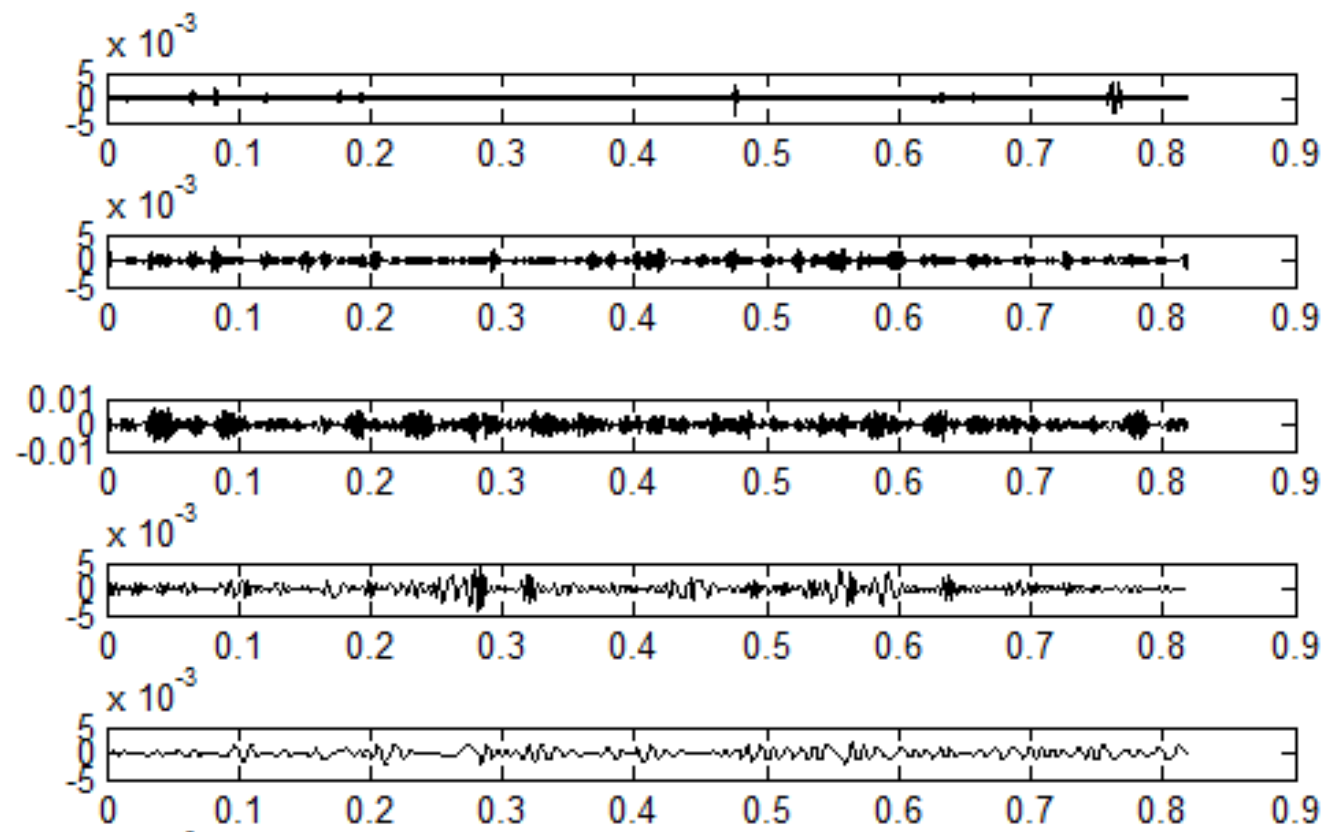

$$
\text { 2. }
$$

Fig. (7). Empirical mode decomposition of tooth break signal. 
Table 1. Sample data of urban railway gearbox operation.

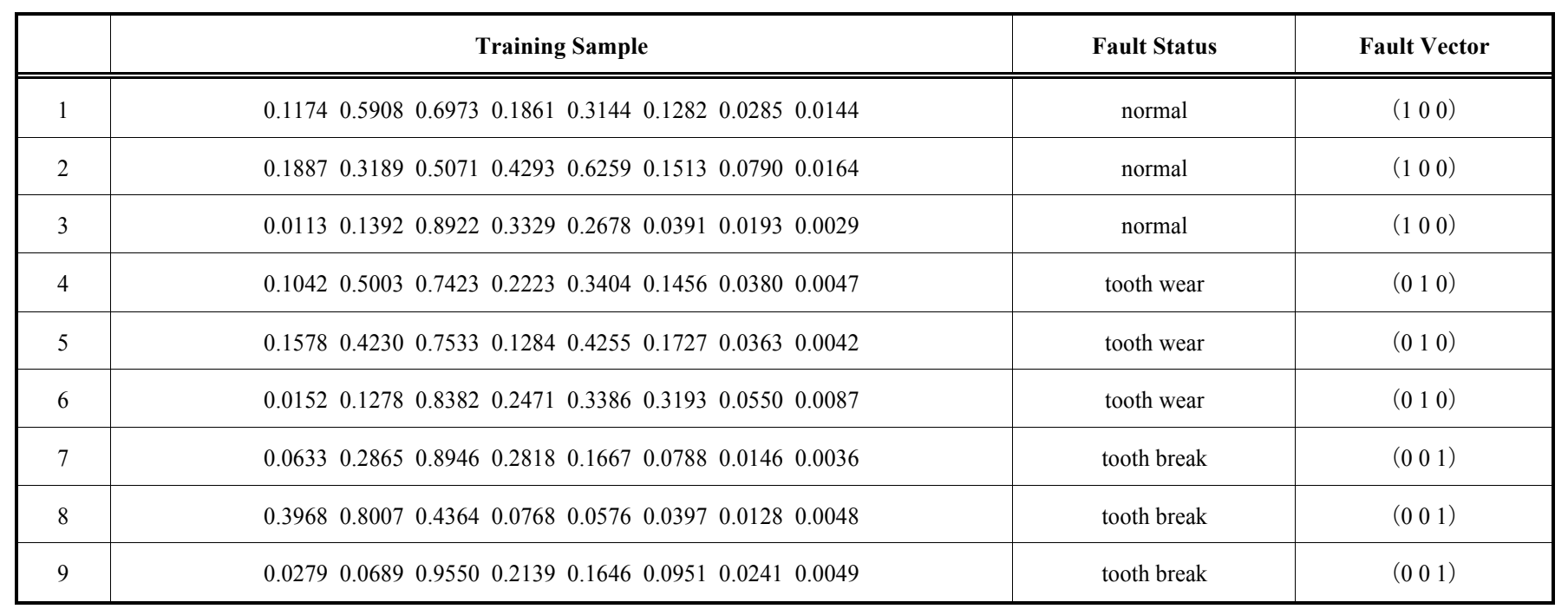

Table 2. Testing data.

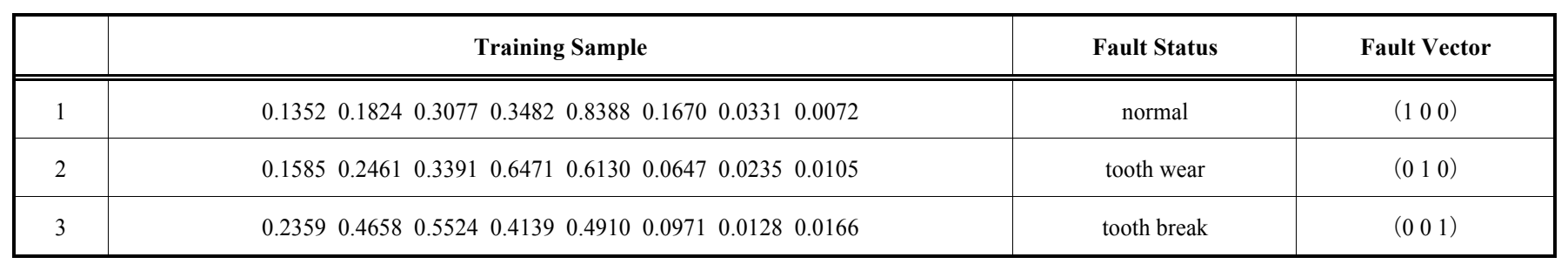

Table 3. Testing results of elman neural network.

\begin{tabular}{|c|c|c|c|}
\hline Fault Status & Fault Vector & Actual Outputs & Testing Results \\
\hline \hline normal & $\left(\begin{array}{lllll}1 & 0 & 0\end{array}\right)$ & $\left(\begin{array}{lllll}0.9326 & 0.0108 & 0.0112\end{array}\right)$ & normal \\
\hline tooth wear & $\left(\begin{array}{lll}0 & 0\end{array}\right)$ & $\left(\begin{array}{lllll}0.0209 & 0.9498 & 0.0279\end{array}\right)$ & tooth wear \\
\hline tooth break & $\left(\begin{array}{llll}0 & 0 & 1\end{array}\right)$ & $\left(\begin{array}{lllll}0.0176 & 0.0255 & 0.9662\end{array}\right)$ & tooth break \\
\hline
\end{tabular}

respectively nonlinear activation function vector of output units and hidden units.

The Elman neural network model is shown in Fig. (1).

\section{EXPERIMENTAL RESULTS AND DISCUSSIONS}

The original time waveforms of vibration signals of normal are provided in Fig. (2), tooth wear are provided in Fig. (3) and tooth break are provided in Fig. (4). The characteristics of the two gearbox running a lot of noise. It is difficult to directly distinguish them by the time domain signals, because the differences among them are very small. Then 6 IMFs are decomposed in each kind of the gearbox vibration signal, and it is shown in Fig. (5-7). After EMD decomposition, the feature vectors are constructed. The method is validated on two datasets, the parts of training data is listed in Table $\mathbf{1}$. The testing data is listed in Table 2.
The network architecture used for fault diagnosis consists of 8 inputs, 8 hidden nodes, and 3 outputs corresponding to 3 respective signals, such as normal signal, tooth wear signal and tooth break signal.

The classifiers based on Elman neural network is trained and tested to identify the fault categories of the railway gearbox. The testing results are shown in Table 3. As shown in this table, the classification of Elman neural network are all close to the corresponding ideal outputs of the examination sample, it is found to be satisfactory and we think that this system can be used in fault diagnosis studies in the future after it is developed.

\section{CONCLUSION}

In this paper, a method for gearbox fault diagnosis is proposed based on a developed signal processing tool named 
EMD and Elman neural network. The testing results show that the provided method can correctly and effectively diagnose railway gearbox faults. Therefore, it can significantly improve the railway system safety and reducing maintenance costs, which will bring huge economic benefits and social benefits. At the same time, the proposed technique provides an effective and attractive method for the rotating machinery fault diagnosis.

\section{CONFLICT OF INTEREST}

The author confirms that this article content has no conflict of interest.

\section{ACKNOWLEDGEMENTS}

This paper was supported by the International Science \& Technology Cooperation Program of China (2014DFR70280).

\section{REFERENCES}

[1] T. Fakhfakh, F. Chaari, M. Haddar, "Numerical and experimental analysis of a gear system with teeth defects", International Journal of Advanced Manufacturing Technology, vol. 25, pp. 542-550, 2005.
[2] Y.J. Li, "EMD-based fault diagnosis for abnormal clearance between contacting components in a diesel engine", Mechanical Systems and Signal Processing, vol. 24, pp.193-210, 2010.

[3] C.-C. Lee, "Robust radial basis function neural networks", IEEE Translated on System, Man, and Cybernetics, Part B, vol. 29, pp. 674-685, 1999.

[4] M.C. Pan, "Using appropriate imfs for envelope analysis in multiple fault diagnosis of ball bearings", International Journal of Mechanical Sciences, vol. 69, pp.114-124, 2013.

[5] N.E. Huang, Z. Shen, S.R. Long, M.C. Wu, H.H. Shah, Q. Zheng, N.C. Yen, C.C. Tung, and H.H. Liu, "The empirical mode decomposition and the Hilbert spectrum for nonlinear and non-stationary time series analysis", Proceedings of the Royal Society of London. A, pp. 903-995, 1998.

[6] G.F. Bin, J.J. Gao and X.J. Li, "Early fault diagnosis of rotating machinery based on wavelet packets-empirical mode decomposition feature extraction and neural network", Mechanical Systems and Signal Processing, vol. 27, pp. 696-711, 2012.

[7] H.M. Liu, S.P. Wang, "Fault diagnosis based on improved elman neural network for a hydraulic servo system", Northern Jiaotong University, 2009.

[8] L. An, N. sepehri, "Leakage fault identification in hydraulic positioning system using extended kalman filter", Proceeding of the 2004 American Conference, Boston, Massachusetts, 2004.

Received: December 15, 2014

(C) Xing et al.; Licensee Bentham Open

This is an open access article licensed under the terms of the Creative Commons Attribution Non-Commercial License (http://creativecommons.org/licenses/by$\mathrm{nc} / 3.0 /$ ) which permits unrestricted, non-commercial use, distribution and reproduction in any medium, provided the work is properly cited. 